Research Article

\title{
Cerebral vascular effects of loading dose of dexmedetomidine: A Transcranial Color Doppler study
}

\author{
Appavoo Arulvelan, Sethuraman Manikandan, Hari Venkat Easwer, \\ Kesavapisharady Krishnakumar
}
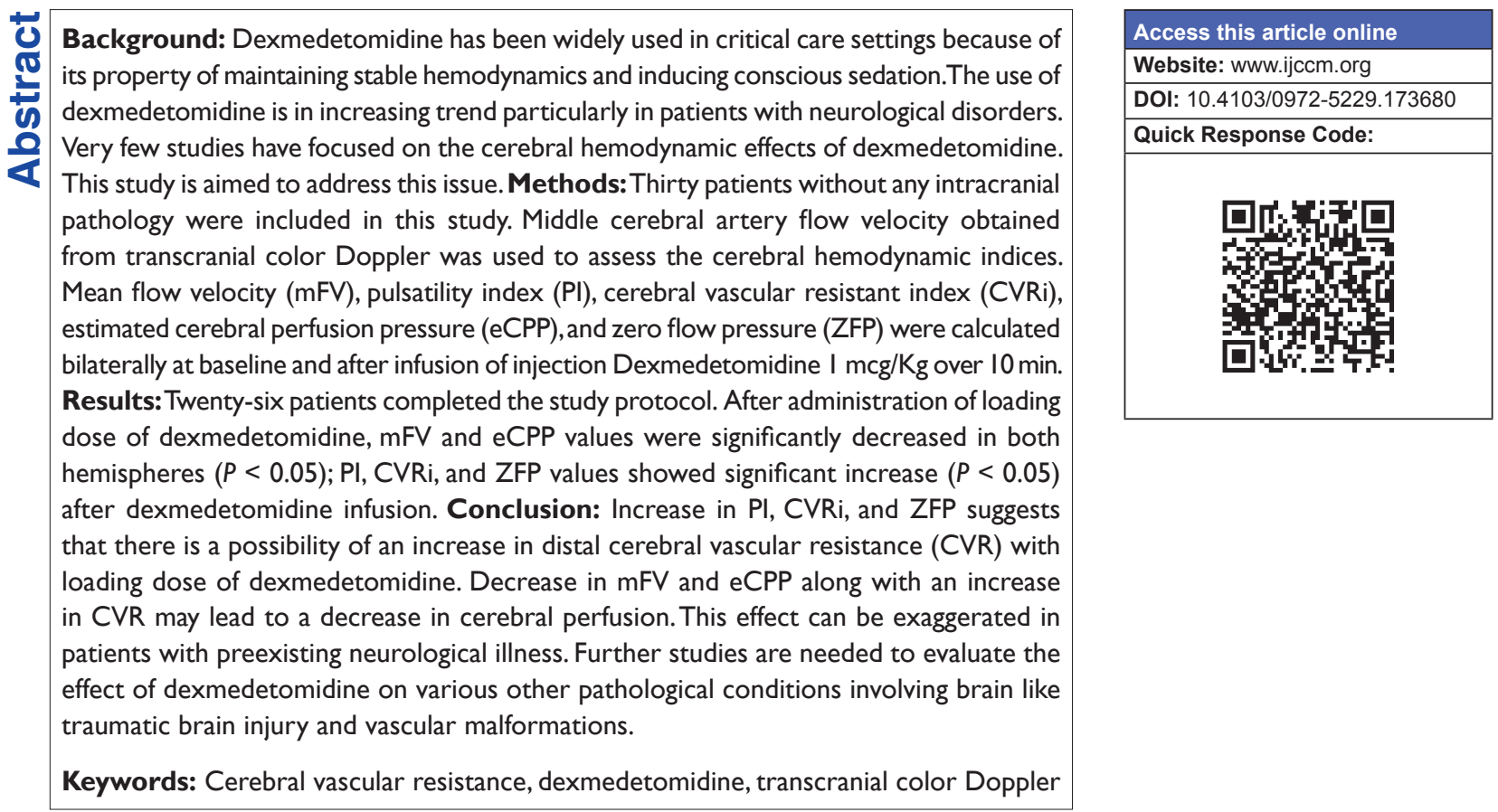

\section{Introduction}

Dexmedetomidine belongs to imidazole subclass of $\alpha_{2}$ adrenoceptor agonists, now widely used in anesthesia and critical care arena. It has been approved for use as sedative and analgesic in the Intensive Care Unit (ICU) setting. It activates presynaptic $\alpha_{2}$ adrenoceptor in the locus coeruleus of pons and inhibits the release of norepinephrine thus leading to sedation and hypnosis. ${ }^{[1]}$ The action of dexmedetomidine is unique as it mimics natural sleep, thus resulting in cooperative form of

From:

Department of Anesthesiology, Sree Chitra Tirunal Institute for Medical

Sciences and Technology, Ulloor, Trivandrum, Kerala, India

Correspondence:

Dr. Appavoo Arulvelan, Sree Chitra Tirunal Institute for Medical Sciences and Technology, Ulloor, Trivandrum - 695 011, Kerala, India.

E-mail: drarulvelan@gmail.com sedation, in which the patients easily transform from sleep to wakefulness when aroused. This characteristic allows for wake up test where the patients are assessed daily for improvement in neurological status, even without stopping sedation thus preventing sympathetic surges during the test. Dexmedetomidine is shown to maintain cognitive and immunologic function in the

This is an open access article distributed under the terms of the Creative Commons Attribution-NonCommercial-ShareAlike 3.0 License, which allows others to remix, tweak, and build upon the work non-commercially, as long as the author is credited and the new creations are licensed under the identical terms.

For reprints contact: reprints@ @edknow.com How to cite this article: Arulvelan A, Manikandan S, Easwer HV, Krishnakumar K.
Cerebral vascular effects of loading dose of dexmedetomidine: A Transcranial Color
Doppler study. Indian J Crit Care Med 2016;20:9-13. 
sleep-deprived states as in the ICU. Clinical side effects of dexmedetomidine described in literature mainly focus on hemodynamic effects such as hypotension and bradycardia. Very few studies have focused on the cerebral hemodynamic effects of dexmedetomidine. ${ }^{[2]}$ Most of the studies on dexmedetomidine and cerebral hemodynamics were conducted upon either animals or in healthy human volunteers with varying doses.

The use of dexmedetomidine is in increasing trend particularly in patients with neurological disorders because of its property of maintaining stable hemodynamics and inducing conscious sedation. In the present study, we tested the cerebral hemodynamic effects of initial loading dose of dexmedetomidine $(1 \mathrm{mcg} / \mathrm{kg}$ over $10 \mathrm{~min})$ with the help of transcranial color Doppler (TCCD) imaging.

\section{Methods}

A pilot study was designed after getting necessary approval from the Institutional Ethical Committee. Thirty American Society of Anesthesiology (ASA) class one patients planned for lumbar discectomy procedure aged between 18 and 40 years were included after obtaining informed consent. Exclusion criteria were: ASA class 2 and above; comorbidities including systemic hypertension and diabetes mellitus, preoperative heart rate $<50 / \mathrm{min}$, the presence of heart block of any degree, and known allergy to dexmedetomidine or other $\alpha_{2}$ agonists.

No premedication was given to the patients. On arrival in the operating room, patients were monitored with electrocardiography (ECG) and pulse oximetry $\left(\mathrm{SpO}_{2}\right)$. Under local anesthesia, intravenous and intra-arterial cannulations were performed. Lactated ringer was given at rate of $4 \mathrm{ml} / \mathrm{kg} / \mathrm{hr}$. After application of nose clip, end tidal $\mathrm{CO}_{2}$ estimation $\left(\mathrm{EtCO}_{2}\right)$ was obtained from mouthpiece and analyzed inside stream monitor (S/5 monitor, GE healthcare, UK). ECG, $\mathrm{SpO}_{2}, \mathrm{EtCO}$, respiratory rate (RR), and invasive blood pressure were monitored and recorded continuously.

TCCD imaging using $2 \mathrm{MHz}$ probe (Vivid-i, GE healthcare, UK) was used to record cerebral blood flow velocities (FV). Through temporal window $\mathrm{M}_{1}$ segment (horizontal segment) of middle cerebral artery (MCA) was identified according to anatomical location, depth of insonation, and direction of flow. After a steady state of FV recording, angle correction was applied so that the angle between linear segment of $M_{1}$ and angle of insonation was less than $15^{\circ}$. Bilateral probes were fixed with custom made headband. FV waveforms were recorded from right and left MCA for consecutive ten cardiac cycles [Figure 1].

Loading dose of dexmedetomidine was given in the dose of $1 \mathrm{mcg} / \mathrm{kg}$ over $10 \mathrm{~min}$ as infusion. During infusion, blood pressure and heart rate were maintained with infusion of lactated ringer solution, injection Ephedrine in $3 \mathrm{mg}$ increments and injection Glycopyrrolate $0.2 \mathrm{mg}$. FV waveforms were recorded from both MCA after $10 \mathrm{~min}$. Once the study protocol was completed, infusion of dexmedetomidine was stopped and routine anesthesia was used for induction and maintenance.

Patients were further excluded if $\mathrm{EtCO}_{2}$ change of more than $2 \mathrm{mmHg}$ from baseline and difficulty in locating MCA with TCCD.

The data collected included demographic data, heart rate, invasive blood pressure, $\mathrm{RR}, \mathrm{SpO}_{2}, \mathrm{EtCO}_{2}$, and bilateral MCA FV at baseline and at the end of the dexmedetomidine infusion.

Mean values of simultaneously measured invasive blood pressure recordings and the outer envelope of time averaged MCA FV recordings during ten consecutive cardiac cycles were used to calculate the following cerebral hemodynamic parameters. ${ }^{[3,4]}$

- Mean flow velocity (mFV)

- Pulsatility index $([\mathrm{PI}]=($ systolic FV - diastolic FV $) /$ mean FV)

- Cerebral vascular resistant index $([\mathrm{CVRi}]=$ mean BP $/$ mean FV)

- $\quad$ Estimated cerebral perfusion pressure $([\mathrm{eCPP}]=$ mean FV $\times[$ mean $\mathrm{BP}-$ diastolic $\mathrm{BP} /$ mean $\mathrm{FV}-$ diastolic FV]) and

- Zero flow pressure $([\mathrm{ZFP}]=$ mean $\mathrm{BP}-\mathrm{eCPP})$.

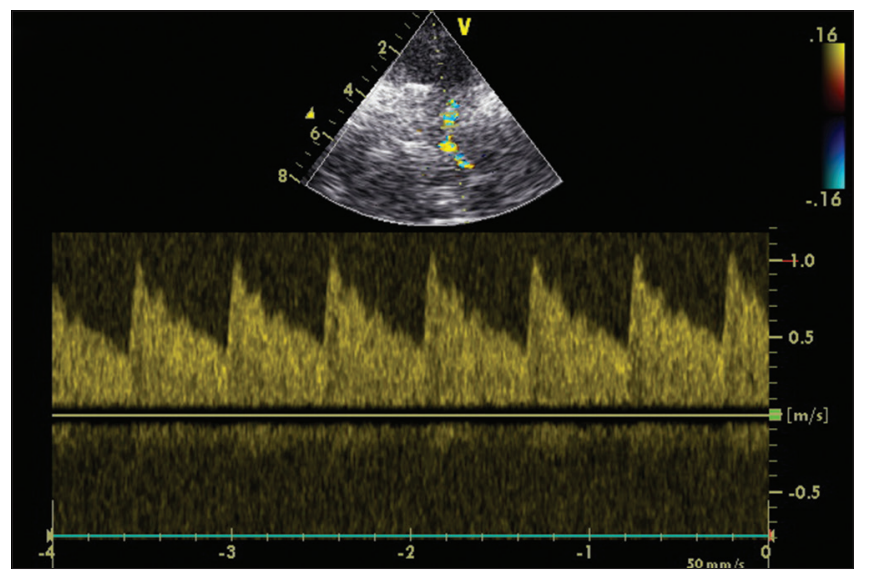

Figure I: Middle cerebral artery flow velocity obtained from transcranial color Doppler imaging 


\section{Statistics}

SPSS version 16 (SPSS, Inc., Chicago, IL, USA, 2007) was used for statistical analysis. Results obtained from the study were expressed in mean $\pm S D$. Changes in vital parameters during dexmedetomidine administration were assessed using one-way ANOVA. Paired $t$-test was used to assess the statistical difference of cerebral hemodynamic parameters ( $\mathrm{mFV}$, PI, CVRi, eCPP, and ZFP) between baseline and after infusion of loading dose of dexmedetomidine. A $P<0.05$ was considered as statistically significant.

\section{Results}

As hemodynamic indices were assessed from both hemispheres, the study groups were subdivided and tabulated as "RIGHT" and "LEFT" representing data from MCA that supplies right and left hemisphere respectively.

Two patients were excluded due to difficulty in localizing MCA, and two patients were excluded because of change in $\mathrm{EtCO}_{2}>2 \mathrm{mmHg}$. Twenty-six patients completed the study protocol. Mean age was $32 \pm 2.7$ years, mean weight was $67 \pm 7.2 \mathrm{~kg}$; 12 were male and 14 were female. Vital parameters that can affect cerebral arterial FV were maintained comparable to baseline values [Table 1].

mFV was significantly decreased in both hemispheres after administration of loading dose of dexmedetomidine $(P<0.001)$. PI and CVRi showed significant increase $(P<0.05)$ after dexmedetomidine loading dose [Table 2]. In both hemispheres, significant decrease in eCPP $(P-0.005 ; P<0.001)$ and significant increase in ZFP $(P<0.001 ; P=0.004)$ were observed [Table 2].

\section{Discussion}

The results of our study showed that the loading dose of dexmedetomidine $(1 \mathrm{mcg} / \mathrm{kg}$ over $10 \mathrm{~min})$ resulted in significant decrease in $\mathrm{mFV}$ and eCPP. We also observed an increase in PI, CVRi, and ZFP after loading dose.
Cerebral vasculature and thus cerebral blood flow is affected by various physiological factors including $\mathrm{paCO}_{2}, \mathrm{PaO}_{2}$, temperature, cerebral metabolic rate, and blood viscosity. ${ }^{[5]}$ These factors are often deranged by disease process or manipulated for therapeutic purposes in anesthesia and critical care environments. Apart from these physiological factors, anesthetic drugs and other vasoactive drugs used in critical care environments can also directly or indirectly affect cerebral blood flow. ${ }^{[6-9]}$ As the use of dexmedetomidine is in increasing trend, knowledge about the cerebral vascular effects will help in the optimal use of this drug with minimal alteration in cerebral hemodynamics.

Transcranial Doppler (TCD) is a simple noninvasive bedside tool to assess the cerebral blood flow pattern. The new development of transcranial color Doppler with angle correction option helps to improve the sensitivity of test results.

\section{Mean flow velocity}

The relationship between TCD-derived FV and actual cerebral blood flow is confounded by various factors such as metabolic state, cerebral vascular resistance (CVR), and cerebral perfusion pressure (CPP). ${ }^{[10,11]}$ In a study by Zornow et al., six healthy volunteers were infused with four different doses of dexmedetomidine. ${ }^{[12]}$ This study showed that hypnotic dose of dexmedetomidine results in a modest decrease in FV corresponding to the increase in dexmedetomidine dosage. Similar results were obtained in our study where the loading dose of dexmedetomidine resulted in a decrease in blood FV. Although mFV derived from TCD is not synonymous with actual cerebral blood flow, studies have shown that the changes in $\mathrm{mFV}$ correlate well with trends of blood flow alteration. ${ }^{[13]}$ As an experimental condition, the blood pressure and $\mathrm{PaCO}_{2}$ were maintained comparable to baseline during the administration of dexmedetomidine, and we have observed decrease in blood FV. However, in patients admitted in critical care units, the baseline blood pressure may be low because of the primary disease or as a side-effect of dexmedetomidine itself. Administration of dexmedetomidine in this scenario can

Table I: Vital parameters

\begin{tabular}{|c|c|c|c|c|c|c|c|}
\hline & Baseline & $2 \mathrm{~min}$ & $4 \mathrm{~min}$ & $6 \mathrm{~min}$ & $8 \mathrm{~min}$ & After DEX & $P$ \\
\hline$\overline{\mathrm{HR}}$ & $74.0(4.8)$ & $72.4(6.3)$ & $75.3(4.5)$ & $72.4(3.2)$ & 75.7 (6.I) & $74.2(5.1)$ & 0.132 \\
\hline MAP & $99.2(3.1)$ & I00.I (4.3) & $97.3(5.3)$ & $96.2(5.2)$ & $97.4(5.1)$ & $95.7(5.0)$ & 0.331 \\
\hline $\mathrm{RR}$ & I4.3(I.I) & I4.3(I.3) & I3.6(I.3) & I3.3(I.0) & $14.5(1.5)$ & I4.3 (I.3) & 0.112 \\
\hline $\mathrm{EtCO}_{2}$ & 34.6 (I.I) & $34.5(1.2)$ & $35.4(1.2)$ & 36.4 (I.I) & $35.5(0.6)$ & $34.4(1.2)$ & 0.221 \\
\hline $\mathrm{SpO}_{2}$ & $99.9(0.4)$ & $99.9(0.4)$ & $98.4(0.5)$ & $99.7(0.3)$ & $99.5(0.5)$ & $99.3(1.0)$ & 0.863 \\
\hline
\end{tabular}

Data shown as mean (SD). After DEX: After dexmedetomidine infusion (I mcg/kg over 10 min); SD: Standard deviation; HR: Heart rate; MAP: Mean arterial pressure; RR: Respiratory rate; $\mathrm{EtCO}_{2}$ : End tidal $\mathrm{CO}_{2} ; \mathrm{SpO}_{2}$ : Oxygen saturation 
Table 2: Cerebral hemodynamic indices

\begin{tabular}{|c|c|c|c|c|c|c|}
\hline & \multicolumn{3}{|c|}{ Right } & \multicolumn{3}{|c|}{ Left } \\
\hline & Baseline & After DEX & $P$ & Baseline & After DEX & $P$ \\
\hline$\overline{\mathrm{mFV}}$ & $57.2(5.2)$ & $50.9(3.5)$ & $<0.00 I^{*}$ & $60.1(3.0)$ & $44.07(2.6)$ & $<0.00 I^{*}$ \\
\hline PI & $0.89(0.1)$ & $1.02(0.06)$ & $0.002 *$ & $0.92(0.03)$ & $1.28(0.04)$ & $<0.00 I^{*}$ \\
\hline CVRi & $1.53(0.2)$ & $\mathrm{I} .85(0.1)$ & $0.003^{*}$ & I.64 (0.I) & $2.15(0.1)$ & $<0.00 I^{*}$ \\
\hline eCPP & $52.46(13.6)$ & $42.3(12.3)$ & $0.005^{*}$ & 47.1 (14.4) & $30.5(7.9)$ & $0.001 *$ \\
\hline ZFP & $45.7(16.1)$ & $58.2(15.7)$ & $<0.00 I^{*}$ & $5 \mathrm{I} . \mathrm{I}(\mathrm{I} 6.8)$ & $64.1(10.5)$ & $0.004 *$ \\
\hline
\end{tabular}

exaggerate the decrease in $\mathrm{mFV}$ and thus compromise on cerebral blood flow.

\section{Pulsatility index and cerebral vascular resistant index}

The normal value for PI is quoted between 0.6 and 0.9. ${ }^{[3]}$ Studies have shown that Gosling PI can be the reliable monitor of cerebral hemodynamic asymmetry and CPP. ${ }^{[14]}$

Stimulation with $\alpha_{2}$ agents such as clonidine and dexmedetomidine has shown to cause vasoconstriction in isolated cerebral vessels and in various animal models. ${ }^{[15-17]}$ Vasoconstrictive property and increase in CVR were also demonstrated in studies where the administration of dexmedetomidine prevented inhalational anesthetics and $\mathrm{CO}_{2}$ induced vasodilatation. ${ }^{[18,19]}$ In a study by Schregel et al., cerebral vascular effects of halothane, alfentanil, and propofol were compared. ${ }^{[20]}$ The PI showed a shortterm increase after alfentanil, decrease after halothane, and strong increase after infusion of propofol. The changes in PI values were consistent with vasodilatory effect of halothane and vasoconstrictory effect of propofol and alfentanil. Increase in PI values obtained from our study suggests the vasoconstrictory effect of dexmedetomidine and possibility of decreased cerebral perfusion. This fact is also supported by the significant decrease in $\mathrm{mFV}$ and increase in CVRi observed in our study.

\section{Cerebral perfusion pressure and zero flow pressure}

$\mathrm{CPP}$ is the net pressure gradient causing blood flow to the brain, and it is calculated as the difference between effective upstream pressure and downstream pressure. ${ }^{[21]}$ It must be maintained within narrow limits because decrease in CPP could cause brain tissue to become ischemic and an increase in CPP can lead to an increase in intracranial pressure (ICP), both detrimental for the survival of neurons. The upstream pressure is usually the mean arterial pressure. As the cranium is a closed cavity, the effective downstream pressure can be central venous pressure (CVP) or ICP or CVR. Thus, a more general concept of ZFP has been introduced, where ZFP is defined as the pressure at which flow in a vessel would cease. Thus, the CPP can be estimated from the difference between MAP and ZFP $[\mathrm{CPP}=\mathrm{MAP}-\mathrm{ZFP}]$.

In our study, significant decrease in eCPP and increase in ZFP were observed. It is shown that, in conditions of normal ICP and CVP, vascular tone may be the dominant component of ZFP. ${ }^{[22]}$ Dexmedetomidine is shown to act as vasoconstrictor in cerebral circulation in various studies. Increase in CVRi values in our study also supports the fact that dexmedetomidine increases cerebral arteriolar tone. Dexmedetomidine is also shown to increase the cerebral venous tone. ${ }^{[23]}$ Thus, it can be explained that the decrease in eCPP and increase in ZFP observed in our study can be due to dexmedetomidine-induced cerebral vasoconstriction and increase in CVR.

"Hypotension" is proven cardiovascular side effect of dexmedetomidine and in our study the "increase in $\mathrm{ZFP}^{\prime \prime}$ is also demonstrated. As the CPP = MAP - ZFP, there is possibility that administration of loading dose of dexmedetomidine can lead to reduction in cerebral blood flow. Apart from CVR, intracranial pathological conditions like cerebral edema, space occupying lesions, blood clots, and elevated venous pressure have shown to increase distal resistance to blood flow. ${ }^{[4,21]}$ When dexmedetomidine is used in these conditions because of vasoconstrictory action, there is a possibility of exaggerated reduction in cerebral blood flow and can be detrimental to neurological outcome.

Inadequate supply of oxygen and glucose to brain leads to cerebral ischemia and excitotoxicity. Adequate supply of substrates depends on adequate perfusion pressure and normal CVR. Results from our study show that after loading dose of dexmedetomidine, there is a possibility of decreased CPP and increased CVR. Our study was done in patients without any systemic comorbid illness and in controlled condition such as maintained blood pressure, maintained $\mathrm{EtCO}_{2}$, and without any other sedative drugs. In ICU setting, these factors may not be under ideal conditions and presence of primary brain pathology may complicate the scenario, further compromising cerebral blood supply.

There are few limitations in our study that are needed to be discussed. In this study, the initial bolus dose of dexmedetomidine $(1 \mathrm{mcg} / \mathrm{Kg}$ over $10 \mathrm{~min})$ was used. Many studies have suggested that the systemic hemodynamic effects such as hypotension and bradycardia can be minimized by omitting the bolus dose 
and initiating the maintenance dose as an infusion. Like the systemic effects, the cerebral vascular effects of the maintenance dose of dexmedetomidine may be different. Thus, further studies are warranted to assess the effect of dexmedetomidine on cerebral hemodynamics with varying maintenance doses. The phenomenon of flow-metabolism coupling with dexmedetomidine is not well defined. ${ }^{[2]}$ Decrease in FV due to metabolic suppression could not be ruled out in our study. We have included patients without intracranial pathology and without any comorbid illness. Studies have shown altered cerebral blood flow and abnormal vascular reactivity in patients with intracranial pathological conditions like infection, trauma, tumor, and vascular malformations. Further adequately powered studies are warranted to address the effect of cerebral hemodynamic effect of dexmedetomidine in pathological conditions involving the brain. TCD-derived parameters correlate well with gross cerebral blood flow. However, the use of monitors such as laser Doppler flowmetry and microdialysis can further help to understand the action of dexmedetomidine at the tissue level.

\section{Conclusion}

In summary, we observed that loading dose of dexmedetomidine $(1 \mathrm{mcg} / \mathrm{kg}$ over $10 \mathrm{~min}$ ) resulted in decrease in FV and estimated CPP even after maintaining blood pressure comparable to baseline values. Increase in PI, CVRi, and ZFP suggests an increase in distal CVR. All these findings collectively suggest the possibility of a decrease in cerebral blood flow after loading dose of dexmedetomidine ( $1 \mathrm{mcg} / \mathrm{Kg}$ over $10 \mathrm{~min}$ ). This effect can be exaggerated in patients with preexisting neurological illness and hemodynamic instability. Further studies are needed to evaluate the effect of dexmedetomidine on various other pathological conditions involving brain like traumatic brain injury and vascular malformations.

\section{Financial support and sponsorship \\ Nil.}

\section{Conflicts of interest}

There are no conflicts of interest.

\section{References}

1. Gerlach AT, Dasta JF. Dexmedetomidine: An updated review. Ann Pharmacother 2007;41:245-52.

2. Bekker A, Sturaitis MK. Dexmedetomidine for neurological surgery. Neurosurgery 2005;57 1 Suppl: 1-10.

3. van Beek AH, Claassen JA, Rikkert MG, Jansen RW. Cerebral autoregulation: An overview of current concepts and methodology with special focus on the elderly. J Cereb Blood Flow Metab 2008;28:1071-85.

4. Hancock SM, Mahajan RP, Athanassiou L. Noninvasive estimation of cerebral perfusion pressure and zero flow pressure in healthy volunteers: The effects of changes in end-tidal carbon dioxide. Anesth Analg 2003;96:847-51

5. Wahl M, Schilling L. Regulation of cerebral blood flow $-\mathrm{A}$ brief review. Acta Neurochir Suppl (Wien) 1993;59:3-10.

6. Dagal A, Lam AM. Cerebral autoregulation and anesthesia. Curr Opin Anaesthesiol 2009;22:547-52.

7. Schlünzen L, Vafaee MS, Cold GE, Rasmussen M, Nielsen JF, Gjedde A. Effects of subanaesthetic and anaesthetic doses of sevoflurane on regional cerebral blood flow in healthy volunteers. A positron emission tomographic study. Acta Anaesthesiol Scand 2004;48:1268-76.

8. Endoh H, Honda T, Ohashi S, Hida S, Shibue C, Komura N. The influence of nicardipine-, nitroglycerin-, and prostaglandin E(1)-induced hypotension on cerebral pressure autoregulation in adult patients during propofol-fentanyl anesthesia. Anesth Analg 2002;94:169-73.

9. Moppett IK, Sherman RW, Wild MJ, Latter JA, Mahajan RP. Effects of norepinephrine and glyceryl trinitrate on cerebral haemodynamics: Transcranial Doppler study in healthy volunteers. Br J Anaesth $2008 ; 100: 240-4$.

10. Ulrich PT, Becker T, Kempski OS. Correlation of cerebral blood flow and MCA flow velocity measured in healthy volunteers during acetazolamide and CO2 stimulation. J Neurol Sci 1995;129:120-30.

11. van der Linden J, Wesslén O, Ekroth R, Tydén H, von Ahn H. Transcranial Doppler-estimated versus thermodilution-estimated cerebral blood flow during cardiac operations. Influence of temperature and arterial carbon dioxide tension. J Thorac Cardiovase Surg 1991; 102:95-102.

12. Zornow MH, Maze M, Dyck JB, Shafer SL. Dexmedetomidine decreases cerebral blood flow velocity in humans. J Cereb Blood Flow Metab 1993;13:350-3.

13. Kontos HA. Validity of cerebral arterial blood flow calculations from velocity measurements. Stroke 1989;20:1-3.

14. Czosnyka M, Richards HK, Whitehouse HE, Pickard JD. Relationship between transcranial Doppler-determined pulsatility index and cerebrovascular resistance: An experimental study. J Neurosurg 1996;84:79-84.

15. Ishiyama T, Dohi S, Iida H. The vascular effects of topical and intravenous alpha2-adrenoceptor agonist clonidine on canine pial microcirculation. Anesth Analg 1998;86:766-72.

16. Ganjoo P, Farber NE, Hudetz A, Smith JJ, Samso E, Kampine JP, et al. In vivo effects of dexmedetomidine on laser-Doppler flow and pial arteriolar diameter. Anesthesiology 1998;88:429-39.

17. Asano Y, Koehler RC, Kawaguchi T, McPherson RW. Pial arteriolar constriction to alpha 2 -adrenergic agonist dexmedetomidine in the rat. Am J Physiol 1997;272(6 Pt 2):H2547-56.

18. Zornow MH, Fleischer JE, Scheller MS, Nakakimura K, Drummond JC. Dexmedetomidine, an alpha 2-adrenergic agonist, decreases cerebral blood flow in the isoflurane-anesthetized dog. Anesth Analg 1990;70:624-30.

19. Fale A, Kirsch JR, McPherson RW. Alpha 2-adrenergic agonist effects on normocapnic and hypercapnic cerebral blood flow in the dog are anesthetic dependent. Anesth Analg 1994;79:892-8.

20. Schregel W, Bredenkötter U, Sihle-Wissel M, Cunitz G. Transcranial Doppler ultrasound: Effects of intravenous anesthetics in neurosurgical patients. Ultraschall Med 1995;16:60-4.

21. White H, Venkatesh B. Cerebral perfusion pressure in neurotrauma: A review. Anesth Analg 2008;107:979-88.

22. Weyland A, Buhre W, Grund S, Ludwig H, Kazmaier S, Weyland W, et al. Cerebrovascular tone rather than intracranial pressure determines the effective downstream pressure of the cerebral circulation in the absence of intracranial hypertension. J Neurosurg Anesthesiol 2000;12:210-6.

23. Ulrich K, Kuschinsky W. In vivo effects of alpha-adrenoceptor agonists and antagonists on pial veins of cats. Stroke 1985;16:880-4. 